\title{
Resolving all atoms of an alkali halide via nanomodulation of the thin NaCl film surface using the $\mathrm{Au}(111)$ reconstruction
}

\author{
K. Lauwaet, ${ }^{1}$ K. Schouteden, ${ }^{1}$ E. Janssens, ${ }^{1}$ C. Van Haesendonck, ${ }^{1}$ P. Lievens, ${ }^{1,}{ }^{*}$ M. I. Trioni, ${ }^{2}$ L. Giordano, ${ }^{3}$ and G. Pacchioni ${ }^{3}$ \\ ${ }^{1}$ Laboratory of Solid-State Physics and Magnetism, KU Leuven, BE-3001 Leuven, Belgium \\ ${ }^{2}$ CNR - National Research Council of Italy, ISTM, Via Golgi 19, I-20133 Milano, Italy \\ ${ }^{3}$ Dipartimento di Scienza dei Materiali, Università di Milano-Bicocca, Via Cozzi 53, I-20125 Milano, Italy
}

(Received 22 January 2012; revised manuscript received 13 April 2012; published 22 June 2012)

\begin{abstract}
We investigated the local influence of the $\mathrm{Au}(111)$ herringbone reconstruction on the properties of thin adsorbed $\mathrm{NaCl}$ films using cryogenic scanning tunneling microscopy (STM) and spectroscopy. Depending on the local hcp versus fcc character of the reconstruction, $\mathrm{NaCl}$ adsorption gives rise to a different shift of the $\mathrm{Au}(111)$ surface state toward the Fermi level, in agreement with ab initio calculations. Such lateral modulation may allow for tunable nanostructuring of thin insulating films, which opens up new perspectives for molecular electronics applications. Furthermore, we demonstrate the simultaneous visualization of both the alkali and the halogen atoms in hcp regions of the $\mathrm{NaCl} / \mathrm{Au}(111)$ surface using a functionalized STM tip. Ab initio calculations relate this simultaneous visualization to the larger electron density in the hcp regions.
\end{abstract}

DOI: 10.1103/PhysRevB.85.245440

PACS number(s): 73.20.-r, 68.35.B-, 68.37.Ef, 68.55.aj

\section{INTRODUCTION}

Nanometer-scale patterned templates with tunable electronic properties are of prime importance in current state-ofthe-art nanotechnologies. The adsorption and self-assembly of functional molecular systems on solid surfaces is a powerful tool to fabricate well-ordered structures that are suitable for future applications in molecular electronics or nanomechanics. ${ }^{1}$ Studies of molecular self-assembly on metal surfaces have shown that such structures can be conveniently grown via self-organization by a proper choice of molecules and substrate. ${ }^{2}$ A notable example is the self-assembly of the fullerene derivative phenyl-C61-butyric-acid-methyl-ester when grown on $\mathrm{Au}(111)$ surfaces, for which there is a preferential adsorption on the fcc areas of the known herringbone reconstruction of $\mathrm{Au}(111)$ surfaces, leading to the growth of molecular nanowires at low coverages. ${ }^{3}$

For the purpose of molecular electronics it is often required that the molecular assemblies are grown on a complementary insulating support rather than directly on a metal support. Thin alkali halide films are considered as an important candidate for this purpose. For example, it has been shown that thin $\mathrm{NaCl}$ films can effectively decouple molecules from the underlying metal support. ${ }^{4}$ Such $\mathrm{NaCl}$ layers have recently also been used successfully for various scanning probe microscopy studies, ${ }^{5-8}$ and self-assembly of adsorbates through intermolecular hydrogen interaction. ${ }^{9}$

Lateral modulation of the interaction between the alkali halide and the underlying metal support may allow for tunable nanostructuring of the thin films and opens up new perspectives for molecular electronics applications. This lateral modulation can be achieved by considering a metal support that exhibits a surface reconstruction. Previous studies are mainly limited to atomically flat supports that do not exhibit a surface reconstruction as for example $\mathrm{Cu}(111)$. The $\mathrm{Au}(111)$ surface is in contrast known for its characteristic herringbone surface reconstruction. In one-half of the reconstructed unit cell, the surface atoms occupy hcp sites, while in the adjacent half they occupy fcc sites. ${ }^{10}$ This reconstruction acts as a superlattice for the $\mathrm{Au}(111)$ Shockley surface state (SS) electrons, thereby creating a modulation in its density. ${ }^{11}$

In this paper we demonstrate that herringbone reconstructed $\mathrm{Au}(111)$ surfaces can be effectively exploited to modulate the surface properties of thin insulating $\mathrm{NaCl}$ films. Relying on scanning tunneling microscopy (STM) and spectroscopy (STS), we reveal that the adsorption of $\mathrm{NaCl}$ causes an upward shift of the $\mathrm{Au}(111) \mathrm{SS}$. This shift is shown to depend spatially on the local $\mathrm{NaCl} / \mathrm{Au}(111)$ interaction, which is modulated by the hcp versus fcc character of the $\mathrm{Au}(111)$ reconstruction. Using a functionalized $\mathrm{Cl}^{-}$terminated STM tip we show that the enhanced electron density at the hcp regions of the modulated $\mathrm{NaCl} / \mathrm{Au}(111)$ interface influence the tip-sample interactions, giving rise to the remarkable simultaneous visualization of both the atomic species of the thin alkali halide bilayer in maps of the local density of states (LDOS). By ab initio calculations we show that the site-specific behavior of the $\mathrm{NaCl}$ indeed stems from the underlying $\mathrm{Au}(111)$ herringbone reconstruction.

\section{EXPERIMENT}

A description of the STM setup and details on the preparation of the $\mathrm{Au}(111)$ surface and $\mathrm{W}$ tips are given elsewhere. ${ }^{12}$ $\mathrm{NaCl}$ (99.999\% purity, Alfa Aesar) is thermally evaporated at $800 \mathrm{~K}$ in a separate setup (pressure in the $10^{-9}$ mbar range) with the substrate kept at room temperature. The sample is transferred to the STM setup by means of an ultrahigh vacuum transport vessel (pressure in the $10^{-10}$ mbar range). All STM measurements are performed at $4.5 \mathrm{~K}$. Image processing is performed by Nanotec WSxM. ${ }^{13}$

\section{RESULTS AND DISCUSSION}

\section{A. $\mathrm{NaCl} / \mathrm{Au}(111)$ interface state}

When deposited on a clean $\mathrm{Au}(111)$ surface at room temperature, $\mathrm{NaCl}$ forms bilayered (100)-terminated islands with perfect nonpolar step edges. ${ }^{9,14}$ Figure 1(a) illustrates the carpetlike growth of $\mathrm{NaCl}$ islands over the steps of the 


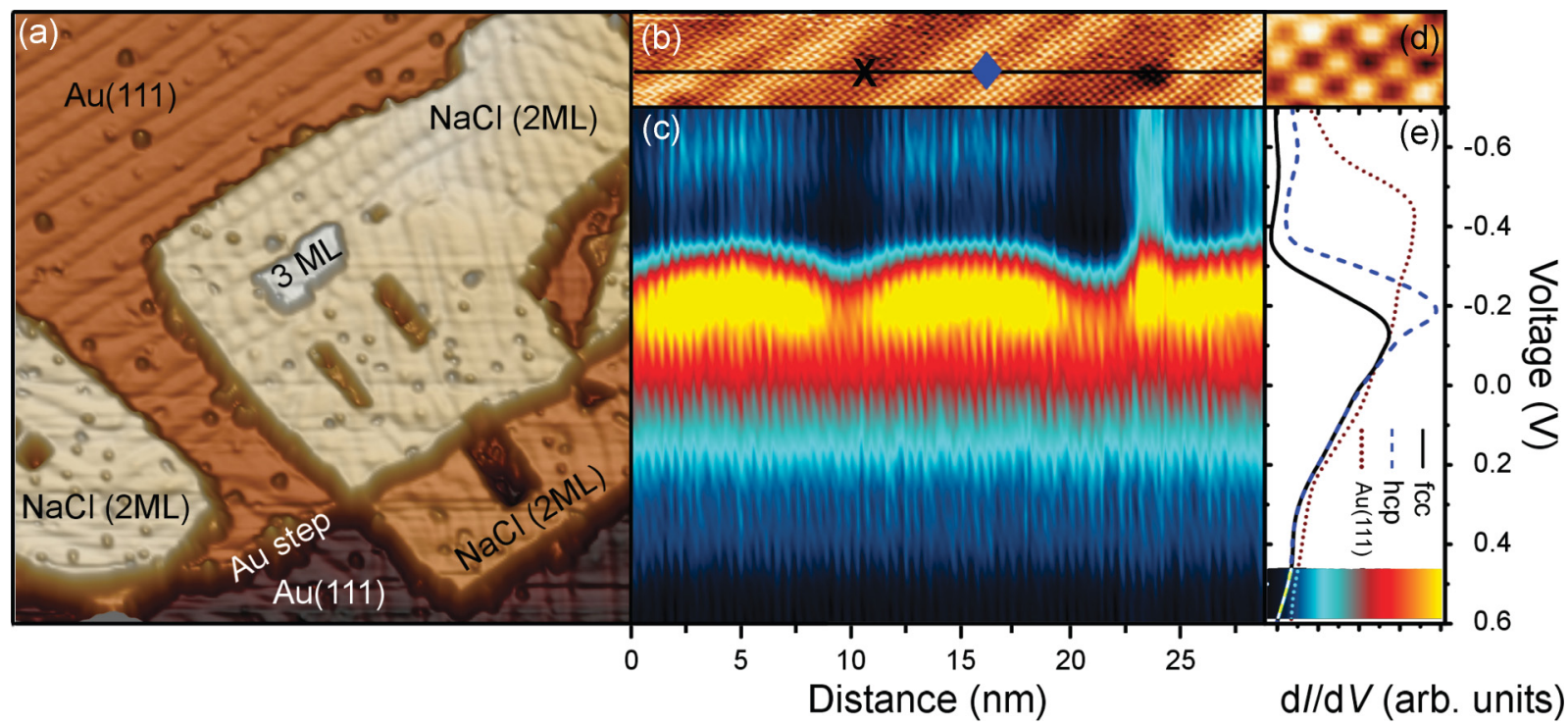

FIG. 1. (Color online) (a) $60 \times 60 \mathrm{~nm}^{2}$ pseudo-three-dimensional (3D) topographical STM image of $\mathrm{NaCl}$ islands grown on Au(111) $(V=1.0 \mathrm{~V} ; I=0.1 \mathrm{nA})$. (b) $30 \times 4 \mathrm{~nm}^{2}$ atomically resolved STM image of trilayer $\mathrm{NaCl}$ grown on $\mathrm{Au}(111)(V=600 \mathrm{mV} ; I=0.6 \mathrm{nA})$. (c) Atomically resolved 2D visualization of $(d I / d V)(V)$ spectra along the black line in (b). The color scale in the figure indicates the density of states; see inset in (e). (d) $1.6 \times 0.9 \mathrm{~nm}^{2}$ atomic resolution closeup view of the trilayer $\mathrm{NaCl}$ island shown in (b). Protrusions correspond to the $\mathrm{Cl}^{-}$ions. (e) $(d I / d V)(V)$ spectra taken on clean $\mathrm{Au}(111)$, and on trilayer $\mathrm{NaCl}$ on the hcp [diamond in (b)] and fcc [cross in (b)] region of the reconstructed $\mathrm{Au}(111)$.

$\mathrm{Au}(111)$ surface. The $\mathrm{Au}(111)$ herringbone reconstruction is not influenced by the $\mathrm{NaCl}$ islands and its corrugation is the same on $\mathrm{NaCl} / \mathrm{Au}(111)$ as on the bare $\mathrm{Au}(111)$. Large patches of trilayer $\mathrm{NaCl}$ were obtained by postannealing to $460 \mathrm{~K}$, which turns the $\mathrm{NaCl}$ from being predominantly bilayered to trilayered. ${ }^{15}$ Figures 1 (b) and 1(d) present typical closeup views of the atomically resolved surface of $\mathrm{NaCl}(3$ $\mathrm{ML}) / \mathrm{Au}(111)$, where the bright protrusions correspond to $\mathrm{Cl}^{-}$. 5,16

In Fig. 1(c) we present an atomically resolved 2D visualization of $(d I / d V)(V)$ curves taken above trilayer $\mathrm{NaCl}$ along the black line indicated in Fig. 1(b). The increased intensity (electron density) around $-250 \mathrm{meV}$ stems from the onset of the newly formed two-dimensional interface state (IS) that originates from the $\mathrm{Au}(111) \mathrm{SS}$ at the $\mathrm{NaCl} / \mathrm{Au}(111)$ interface. Remarkably, the onset energy $E_{0}$ of the IS appears to be spatially dependent on the $\mathrm{Au}(111)$ herringbone reconstruction. From the spectra in Fig. 1(e) it can be seen that the IS is shifted by an extra $60 \mathrm{meV}$ toward the Fermi level in the fcc region when compared to the hcp region. The ridges provide a smooth transition of the IS shift between the two regions of the reconstruction. A similar modulation of the IS by the $\mathrm{Au}(111)$ herringbone reconstruction is observed for bilayered $\mathrm{NaCl}$. On pristine $\mathrm{Au}(111)$, however, the delocalized SS electrons respond to the reconstruction mainly by spatially transferring state density near $E_{0}$ of the $\mathrm{SS}$ around $-480 \mathrm{meV}$, but there is no difference in the value of $E_{0}$ of the SS for the fcc and hcp regions. ${ }^{11}$

To understand the origin of the observed shift and modulation of $E_{0}$, we have performed $a b$ initio calculations for the $\mathrm{NaCl} / \mathrm{Au}(111)$ system. The complexity of the system, associated with (i) the long-range herringbone reconstruction and (ii) the incommensurate growth of the square $\mathrm{NaCl}$ lattice on the hexagonal $\mathrm{Au}(111)$ surface, makes a full $a b$ initio treatment of the system unfeasible. The IS shift analysis was therefore performed in three steps, here described briefly (see the Appendix for a more detailed description). First, the clean $\mathrm{Au}(111)$ surface is described using the "embedding method" approach to model the potential along the $z$ coordinate normal to the surface. ${ }^{17,18}$ Key quantities such as the bulk band projected gap at $\bar{\Gamma}, E_{0}$ of the $\mathrm{Au}(111) \mathrm{SS}$, and the work function of the $\mathrm{Au}(111)$ surface are correctly described by this model. Second, the more attractive potential in the hcp region is modeled by considering the potential change induced in the surface region upon a $3 \%$ compression of the interatomic distances. To simulate this compression $a b$ initio perturbing potentials are employed. A downward shift of $48 \mathrm{meV}$ (i.e., $16 \mathrm{meV}$ for each unit of percentage of compression) was found, which is consistent with the attractive potential of $25 \mathrm{meV}$ for the hcp region estimated by Chen et al. ${ }^{11}$ Third, the adsorbed $\mathrm{NaCl}$ bilayer is simulated by potential wells computed $a b$ initio and fitted by an analytical model potential. The $\mathrm{NaCl}$ film causes the $\mathrm{Au}(111) \mathrm{SS}$ to become an IS that is localized in the region between the metal and the $\mathrm{NaCl}$ film. Although the potential due to the overlayer is attractive, the ionic film acts as a repulsive potential for the $\mathrm{SS}$, implying that the IS is less bound. This can be understood considering the potential barrier generated by the insulating gap. In some energy regions the electronic states cannot penetrate. Consequently, the potential well "felt" by the SS is narrower and the bound state shifts from $-480 \mathrm{meV}$ to $-220 /-270 \mathrm{meV}$.

The model potentials of pristine $\mathrm{Au}(111)$ and of the $\mathrm{NaCl} / \mathrm{Au}(111)$ system in the fcc region are presented in Fig. 2, together with the calculated square moduli of the SS and IS in the fcc region. By taking the potential of the adsorbed insulating layer explicitly into account, we find that the IS 


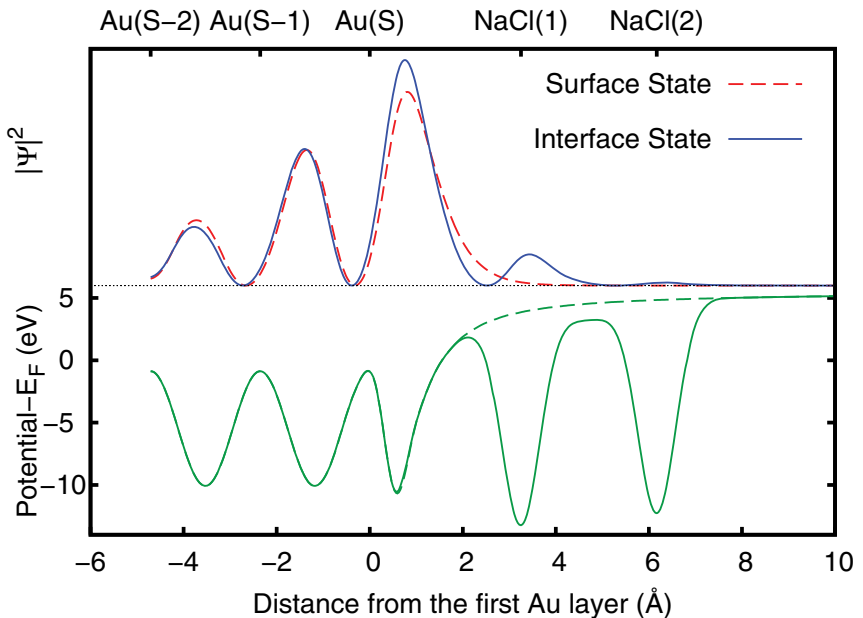

FIG. 2. (Color online) Lower panel: model potentials for $\mathrm{Au}(111)$ (dashed line) and $\mathrm{NaCl}(2 \mathrm{ML}) / \mathrm{Au}(111)$ (solid line) in the fcc region. Upper panel: squared moduli of the wave functions for the $\mathrm{Au}(111)$ $\mathrm{SS}$ and $\mathrm{NaCl} / \mathrm{Au}(111)$ IS in the fcc region.

wave function exhibits maxima at the positions of the two $\mathrm{NaCl}$ layers, unlike in earlier work where the $\mathrm{NaCl}$ is taken into account only via its static dielectric constant. ${ }^{5}$ The calculated values for $E_{0}$ of the IS in the different regions of the $\mathrm{Au}(111)$ reconstruction are listed in Table I. The good agreement between the theoretical and experimental values of $E_{0}$ in both the fcc and hcp regions demonstrates that the proposed model potential captures the physics involved in the IS shift. As the IS wave function is already damped after two $\mathrm{NaCl}$ layers, the calculated shift for the trilayer (see Table I) is essentially the same as for the bilayer, suggesting that the experimentally found small difference may be related to a difference in the structural parameters of bilayer and trilayer $\mathrm{NaCl}$ films.

\section{B. Simultaneous imaging of the $\mathrm{Na}$ and $\mathrm{Cl}$ atoms}

The above results clearly demonstrate that the reconstruction of the $\mathrm{Au}(111)$ surface gives rise to the spatially modulated electronic properties of the $\mathrm{NaCl} / \mathrm{Au}(111)$. This modulation allows us to investigate the influence of the local electronic properties of the $\mathrm{NaCl} / \mathrm{Au}(111)$ system on the site-specific interaction between the $\mathrm{NaCl}$ and an adsorbate. For this purpose, we investigated the local interaction between the alkali halide film and a $\mathrm{Cl}^{-}$terminated STM tip. Such a tip was obtained simply by scanning the bilayer $\mathrm{NaCl}$ surface in close proximity until a change of the microscope tip apex

TABLE I. Theoretical and experimental values of the onset energy $E_{0}$ (in $\mathrm{meV}$ ) of the $\mathrm{NaCl} / \mathrm{Au}(111)$ IS for both fcc and hep $\mathrm{Au}(111)$ reconstructed regions. Experimental values are determined from $(d I / d V)(V)$ curves following the geometrical procedure given in Ref. 19. The experimental $E_{0}$ of the pristine $\mathrm{Au}(111) \mathrm{SS}$ is $-480 \pm 10 \mathrm{meV}$ for both regions.

\begin{tabular}{lcccccc}
\hline \hline & \multicolumn{2}{c}{ Experimental } & & \multicolumn{2}{c}{ Theoretical } \\
\cline { 2 - 3 } & fcc & hcp & & fcc & hcp \\
\hline $\mathrm{NaCl}(2 \mathrm{ML})$ & $-220 \pm 20$ & $-270 \pm 20$ & & -211 & -267 \\
$\mathrm{NaCl}(3 \mathrm{ML})$ & $-230 \pm 20$ & $-290 \pm 20$ & & -211 & -268 \\
\hline \hline
\end{tabular}

was observed..$^{20}$ Local variations of the interaction between tip and sample were measured by mapping the LDOS. ${ }^{21}$ Figures 3(a) and 3(b) present a topographical STM image and the corresponding LDOS map of a bilayer $\mathrm{NaCl}$ island recorded with a functionalized STM tip. In the topography image in Fig. 3(a) only one of the atomic species of $\mathrm{NaCl}$ is revealed. Remarkably, the LDOS map in Fig. 3(b) reveals a different resolution on the fcc and the hcp regions: whereas only one of the atomic species is observed in the fcc regions (similar to the topography image), both atomic species are visualized in the hcp regions (in contrast to the topography image). Both atoms are also resolved on the "ridges" of the herringbone reconstruction, which provide a transition region from the fcc to the hep configuration. This is the first observation of both atomic species of an alkali halide in real space by scanning probe experiments. Previously, imaging of both ionic sublattices has been achieved on $\mathrm{TiO}_{2}{ }^{22}$ Our result demonstrates that one can modulate the surface properties of an alkali halide by growing the alkali halide on a patterned support.

Now the question arises whether the observed simultaneous imaging of the $\mathrm{Na}$ and $\mathrm{Cl}$ atoms is related to the inherent LDOS of the $\mathrm{NaCl} / \mathrm{Au}(111)$ system only or if specific tipsample interactions play a prominent role as well? To answer this question, we calculated the electronic structure of the $\mathrm{NaCl} / \mathrm{Au}(111)$ system with density functional theory (DFT) and simulated LDOS maps neglecting possible tip effects. If the observed simultaneous imaging would not be related to specific tip-sample chemical interactions, these simulated LDOS maps must be able to describe the images in Figs. 3(a) and $3(\mathrm{~b}){ }^{23}$ If not, the simultaneous imaging is (at least partially) related to tip-sample interactions. ${ }^{24}$

DFT calculations are performed with the exchangecorrelation functional proposed by Perdew, Burke, and Ernzerhof. $^{25}$ We constructed a model of the system with a coincidence structure, obtained by superposing a $(2 \times 2)$ $\mathrm{NaCl}$ unit cell on a $\left(\begin{array}{ll}3 & 1 \\ 1 & 3\end{array}\right)$ superstructure of the $\mathrm{Au}(111)$ substrate. This coincidence structure presents a residual strain of about 5\%, which is accommodated in the Au(111) substrate. Moreover, the angle of the substrate unit cell is changed from $82^{\circ}$ to $90^{\circ}$ to match the square symmetry of the $\mathrm{NaCl}$ film. We have chosen to accommodate the distortion on the metallic substrate and to keep the $\mathrm{NaCl}$ undistorted to avoid artificial rumpling in the deposited film, although we verified that the results obtained by deforming the $\mathrm{NaCl}$ are essentially the same. The metal surface is modeled by a five atomic layers thick slab. The atomic coordinates of the Au slab are relaxed in $z$ direction in order to accommodate the above-mentioned strain and are then kept fixed for the $\mathrm{NaCl} / \mathrm{Au}$ combined system. The $\mathrm{NaCl}$ coordinates are then relaxed in $z$ direction and the $\mathrm{NaCl}$ in-plane lattice parameter is fixed at $3.91 \AA$, the average of the optimized lattice parameters of unsupported $\mathrm{NaCl}$ bilayer and trilayer.

We have included an estimate of the van der Waals interactions, not present in DFT theory, with the pair-wise force field as implemented in the DFT-D2 method of Grimme. ${ }^{26}$ The model accounts for the experimentally measured work function reduction of $1.1 \mathrm{eV}$ with respect to the $\mathrm{Au}(111)$ surface, ${ }^{27}$ estimated from the energy shift of the $n=2$ 

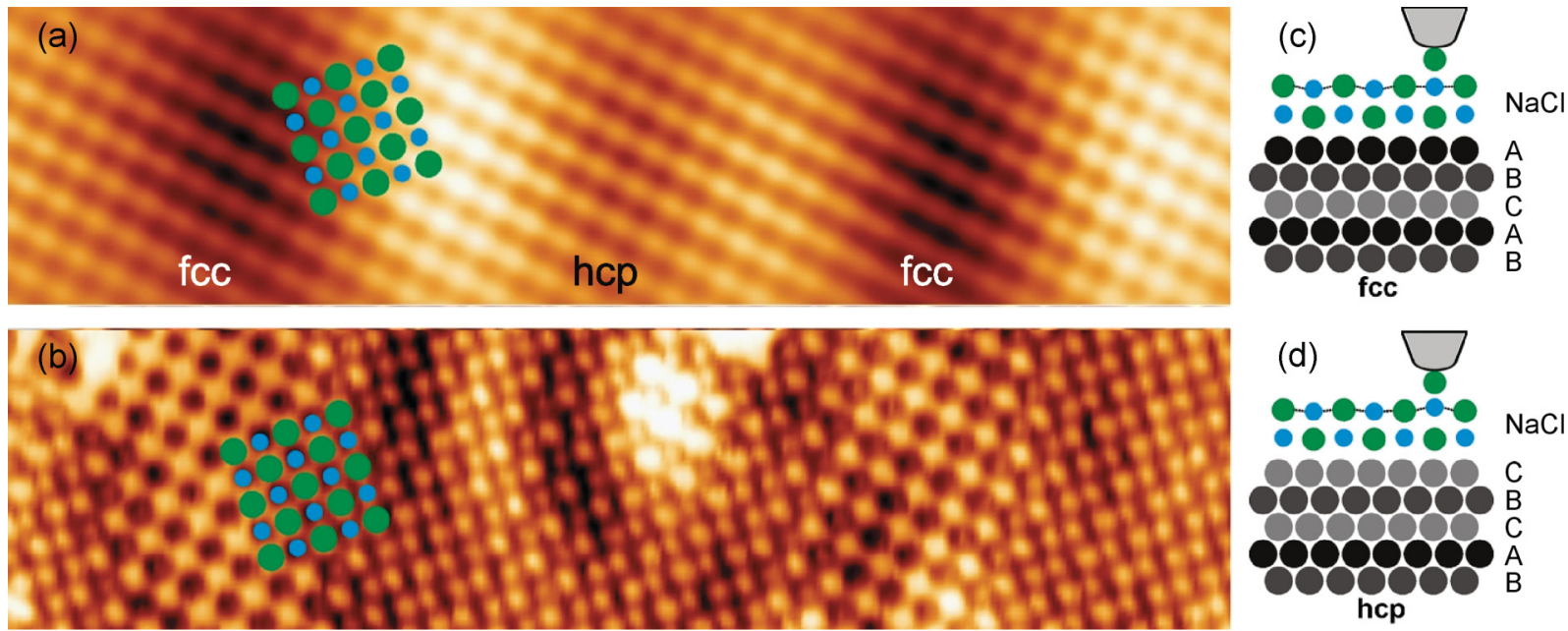

FIG. 3. (Color online) (a) $2 \times 13 \mathrm{~nm}^{2}$ topographical STM image (after applying a Fourier filter) and (b) corresponding LDOS map (raw data) of $\mathrm{NaCl}(2 \mathrm{ML}) / \mathrm{Au}(111)(V=600 \mathrm{meV} ; I=0.4 \mathrm{nA})$. Whereas only the $\mathrm{Cl}$ atoms are resolved in the topographic image (a) as well as in the fcc region of the LDOS map (b), both the $\mathrm{Na}$ and the $\mathrm{Cl}$ atoms are visible in the hcp region of the LDOS map (b). A model of the $\mathrm{NaCl}$ lattice is added as a guide for the eye (Cl: large dots; Na: small dots). (c) and (d) Schematic representation of bilayer $\mathrm{NaCl}$ on fcc and hcp $\mathrm{Au}(111)$, respectively, that are imaged by a Cl terminated STM tip. Note that the bulk Au stacking remains intact, while only the stacking of the top atomic layer is different in the fcc and hcp regions. For clarity reasons the $\mathrm{NaCl}$ rumpling is not drawn to scale.

image state. The interface coincidence structure does not allow one to model the hcp region of the $\mathrm{Au}(111)$ by a compression of the interatomic distances. Instead, the $\mathrm{Au}(111)$ surface reconstruction is taken into account by considering an artificially reduced $\mathrm{NaCl} / \mathrm{Au}(111)$ interface distance in the hcp region (by $0.02 \mathrm{~nm}$ ), which gives rise to an enhanced electron density in this region, in agreement with the $(d I / d V)(V)$ curves in Fig. 1(e). Upon atomic relaxation we find a reduced $\mathrm{NaCl}$ rumpling in the hcp region (about $6.3 \mathrm{pm}$, with the $\mathrm{Cl}$ relaxing outward) compared to the fcc region (about $7.6 \mathrm{pm}$ ). This effect is schematically illustrated in Figs. 3(c) and 3(d). The computed STM images for both regions (fcc and hcp) always show the $\mathrm{Cl}$ atom only, in agreement with the experiments [Fig. 3(a)]. However, the simulated LDOS maps also show the $\mathrm{Cl}$ atom only and hence cannot account for the observed imaging of both types of atoms. It must be noted that Figs. 3(a) and 3(b) are recorded at the same feedback settings that enabled the STM tip to pick up a $\mathrm{Cl}$ atom, implying a small tip-sample distance. It is thus reasonable that the STM tip, neglected in the simulations, plays indeed a key role in the imaging. 23,24

Hence we verified the role of the functionalized STM tip (i.e., terminated by a $\mathrm{Cl}^{-}$ion) for the visualization of both atomic species in the hcp regions of the $\mathrm{NaCl}$ surface in LDOS maps by electrostatically attracting the $\mathrm{Na}^{+}$ion above the surface. For this purpose, we calculated the energy cost of lifting a $\mathrm{Na}$ atom from the $\mathrm{NaCl}$ layer for both hcp and fcc $\mathrm{NaCl} / \mathrm{Au}(111)$, which allows its detection in LDOS maps. We find that the average energy needed to lift a $\mathrm{Na}$ atom to the level of the mean $\mathrm{Cl}$ plane is $20 \%$ larger for the fcc region than for the hcp region (of the order of $5 \mathrm{meV}$ ). The (additional) rumpling of the $\mathrm{NaCl}$ surface induced by a negative ion on the STM tip ape ${ }^{28}$ is schematically illustrated in Figs. 3(c) and 3(d) and can explain the enhanced $\mathrm{Na}$ signal at hcp regions in the experimental LDOS maps. Furthermore, our calculations show that the DOS at the $\mathrm{Na}(\mathrm{Cl})$ sites increases (decreases) with increasing energy above the Fermi level. This implies that simultaneous visualization of both atomic species is most pronounced in LDOS maps [Fig. 3(b)], i.e., at positive bias voltages, while it is less pronounced or even not discernible in topography images [Fig. 3(a)].

\section{CONCLUSIONS}

In summary, by growing $\mathrm{NaCl}$ on a $\mathrm{Au}(111)$ surface we are able to study the interaction between a stiff insulating material and a reconstructed surface. This interaction is found to modulate the newly formed IS that is confined by the $\mathrm{NaCl}$ potential barrier, which acts differently in the hcp and fcc regions of the $\mathrm{Au}(111)$ reconstruction. Using a functionalized STM tip, the enhanced electron density in the hcp regions allows us to resolve both species of the alkali halide. The $\mathrm{NaCl} / \mathrm{Au}(111)$ system opens new perspectives for the templated growth of self-organized molecules.

\section{ACKNOWLEDGMENTS}

This research was supported by the Research Foundation Flanders (FWO, Belgium) and the Flemish Concerted Action (GOA) research program. K.L. acknowledges the Institute for the Promotion of Innovation through Science and Technology in Flanders (IWT-Vlaanderen) for additional financial support, while K.S. acknowledges the FWO for additional financial support. We thank the Regione Lombardia and CILEA (LISA initiative) Consortium for a CPU grant.

\section{APPENDIX: THEORETICAL MODELING OF THE INTERFACE STATE MODULATION OF NaCl(2-3 ML)/Au(111)}

The clean $\mathrm{Au}(111)$ surface and the $\mathrm{NaCl}$ film are described by a model potential depending on the $z$ coordinate only, 


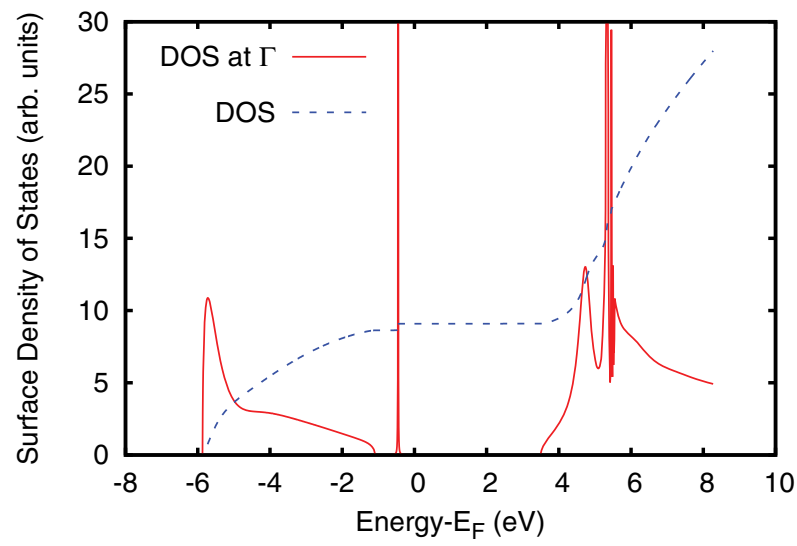

FIG. 4. (Color online) Surface density of states for the $\mathrm{Au}(111)$ surface. DOS at $\bar{\Gamma}$ (solid line) and DOS integrated over the surface Brillouin zone (dashed line). The region considered for the spatial integration contains two gold layers plus the vacuum.

normal to the surface. We use a phenomenological potential ${ }^{18}$ to describe the clean $\mathrm{Au}(111)$ surface. Ab initio perturbing potentials were then employed to simulate the surface reconstruction and the $\mathrm{NaCl}$ adsorption. A similar model was used to describe the upward shift of the surface state in Ref. 29, although our model takes explicitly into account the potential of the insulating layer.

\section{Clean $\mathrm{Au}(111)$}

The model potential describing the $\mathrm{Au}(111)$ surface has been proposed in literature some years ago by Chulkov et al. ${ }^{18}$ and is reported as dashed line in the lower panel of Fig. 2. This potential has been built in order to reproduce some experimental features which play a role in our experimental observations: the amplitude and energy position of the surface projected bulk band gap, the energy of the Shockley surface state, and the work function of the gold metal surface.

Following the embedding method proposed by Inglesfield, ${ }^{17}$ we use semi-infinite boundary conditions to solve the Schrödinger equation, obtaining results close to the experimental ones (see Table II).

In Fig. 4 the surface density of states (DOS) at $\bar{\Gamma}$ (solid line) for the $\mathrm{Au}(111)$ clean surface is presented. The surface projected bulk band occupies the higher binding energy part of the spectrum. The model potential is able to describe only the

TABLE II. Parameters for the $\mathrm{Au}(111)$ surface. Energies in $\mathrm{eV}$ with respect to the Fermi energy for all data, except for the image state which is given with respect to the vacuum.

\begin{tabular}{lccr}
\hline \hline & Experiment & Theory in Ref. 18 & This work \\
\hline Lower gap edge & -1.0 & -1.0 & -1.09 \\
Upper gap edge & 3.6 & 3.6 & 3.51 \\
Shockley SS & $-0.48^{\mathrm{a}}$ & -0.47 & -0.47 \\
First image state & $-0.6^{\mathrm{b}}$ & -0.80 & -1.01 \\
\hline \hline
\end{tabular}

a Some other values are reported in literature: $-0.50,{ }^{30}-0.35,{ }^{31}$ $-0.47,{ }^{32}$ and $-0.41 .^{33}$

${ }^{\mathrm{b}}$ As taken from Ref. 30, but some other values are reported in literature: $-0.42^{34}$ and $-0.8 .^{35}$

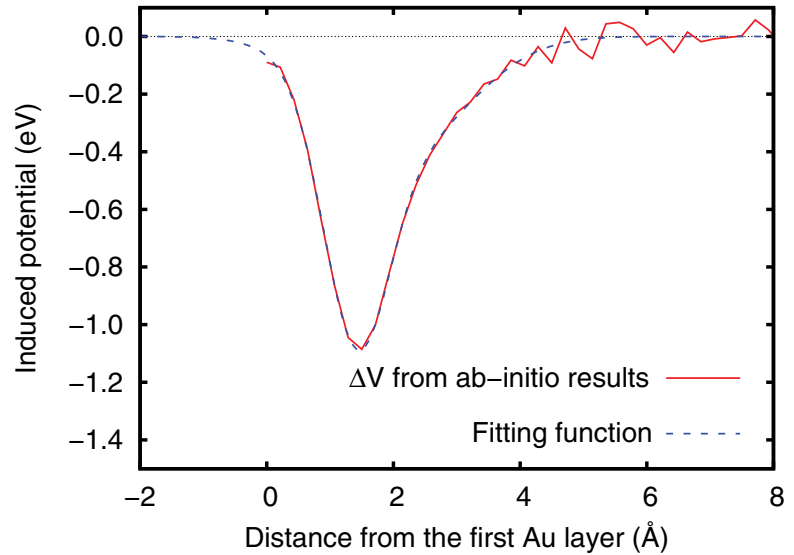

FIG. 5. (Color online) Potential induced by a 5\% compression of the interatomic distance.

$s$ - $p$-like band. The $d$ band is absent, but this does not play any role for the experimental results considered here. In the gap, extending from -1.09 to $3.51 \mathrm{eV}$, the Shockley surface state at $-0.468 \mathrm{eV}$ is clearly visible. The upper gold bulk band starts at $3.51 \mathrm{eV}$ and the image state series appears in this band near the vacuum level. The work function for this system is $5.55 \mathrm{eV}$. The dashed line in Fig. 4 represents the DOS integrated over the surface Brillouin zone. As expected for the surface state, the DOS shows a steplike behavior which can be probed by scanning tunneling spectroscopy.

\section{Effects due to surface reconstruction}

All the $a b$ initio calculations were performed within the generalized gradient approximation of DFT as proposed by Perdew, Burke, and Ernzerhof (GGA-PBE) ${ }^{25}$ A five layers $\mathrm{Au}$ slab was considered to determine the effective potential in the surface region. After a structural relaxation with the theoretical bulk lattice parameter $(4.17 \AA)$, the resulting structure has been scaled to the experimental one $(4.08 \AA){ }^{36}$ The corresponding surface interatomic distance amounts to $2.88 \AA$. To address the influence of the altered in-plane bond length along the $\mathrm{Au}(111)$ reconstruction, simulations were also performed using shorter interatomic distances while keeping the perpendicular coordinates fixed. In Fig. 5 the lateral average of the potential induced in the surface region caused by a $5 \%$ compression of the interatomic distance is presented. More explicitly we present the quantity

$$
\begin{aligned}
\Delta V_{\mathrm{e} f f}(z)= & \frac{1}{S} \int_{S} d x d y V_{\mathrm{eff}}^{5 \%}(x, y, z) \\
& -\frac{1}{S^{\prime}} \int_{S^{\prime}} d x d y V_{\mathrm{eff}}^{0}(x, y, z),
\end{aligned}
$$

where $S\left(S^{\prime}\right)$ is the surface unit cell and $V_{\text {eff }}$ is the local part of the Kohn-Sham effective potential. We verified that $\Delta V_{\text {eff }}(z)$ depends linearly on the compression. By adding this quantity to our model potential for the $\mathrm{Au}(111)$ surface we are able to simulate in a very simple way different regions where the gold atoms arrange themselves according to a hcp or fcc stacking.

The induced potential is attractive and it should increase the binding energy of the surface state. In fact, we found a downward shift of $16 \mathrm{meV}$ for each unit of percentage of 


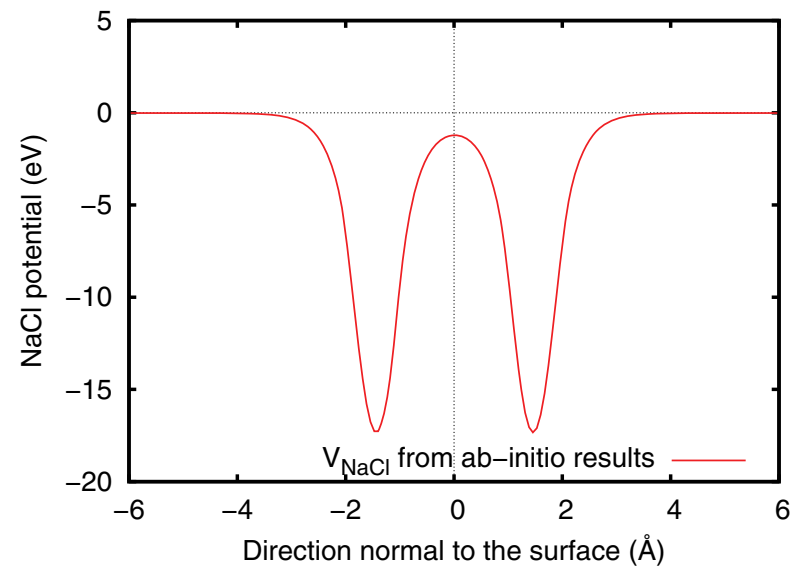

FIG. 6. (Color online) Lateral average of the ab initio effective potential for the $\mathrm{NaCl}$ bilayer.

compression. There is no experimental verification possible for this quantity, but as a first approximation it can be compared to the attractive potential of $25 \mathrm{meV}$ for the hcp region proposed in Ref. 11.

\section{Adsorption of $\mathrm{NaCl}$}

In order to simulate the adsorption of a bilayer of $\mathrm{NaCl}$ we added in front of the $\mathrm{Au}(111)$ surface a double well potential computed ab initio and fitted by an analytical model potential. In Fig. 6 we present the lateral average of the effective potential computed within DFT.

The interface distance has been arbitrarily fixed to $3.2 \AA$ which is comparable to the sum of the covalent radius of $\mathrm{Au}$ and the ionic radius of $\mathrm{Cl}^{-}$. As will be discussed below, the results depend on this distance.

For the $\mathrm{NaCl}$ interlayer distance we take the computed equilibrium distance of the $\mathrm{NaCl}$ bilayer, i.e., $2.828 \AA$ ( $2.815 \AA$ is the experimental equilibrium distance for bulk $\mathrm{NaCl}$ ). The model potential representing the $\mathrm{NaCl}$ bilayer adsorbed on $\mathrm{Au}(111)$ is presented as a solid line in the lower panel of Fig. 2. The presence of the $\mathrm{NaCl}$ bilayer causes an upward shift of the pristine Shockley state, which is now better described as an IS localized in the region between the metal and the $\mathrm{NaCl}$ film.

The IS is now $257 \mathrm{meV}$ less bound, i.e., its energy is $-211 \mathrm{meV}$. Also the phase shift model has been used by Repp et al. to understand this phenomenon. ${ }^{29}$ In Ref. 29 the upward shift of the surface state is attributed to the weaker image potential due to the polarization of the adsorbed dielectric.

A comparison between the Shockley surface state and the IS is presented in the upper panel of Fig. 2. The narrower shape of the principal maximum of the IS indicates a stronger confinement of this state.

\section{Effect on the interface state due to surface reconstruction}

The effect of the compression of the $\mathrm{Au}(111)$ surface, simulating the hcp region, can be added to the previously calculated effective potential. With this additional attractive perturbation the IS is located at $-0.267 \mathrm{meV}$, i.e., more bound by $56 \mathrm{meV}$ for a compression of 3\%. All the previously reported results are obtained for a fixed $\mathrm{NaCl}-\mathrm{Au}(111)$ distance. Indeed, a variation of this distance modifies the binding energy of the IS: we estimate a downward shift by about $45 \mathrm{meV}$ for an increase in distance of $0.1 \AA$. In a similar way, a rearrangement of the overlayer, i.e., a rumpling of the $\mathrm{NaCl}$ surface layer, can influence the results as well.
*Peter.Lievens@fys.kuleuven.be; http://fys.kuleuven.be/vsm/

${ }^{1}$ J. V. Barth, Annu. Rev. Phys. Chem. 58, 375 (2007).

${ }^{2}$ J. A. A. W. Elemans, S. B. Lei, and S. De Feyter, Angew. Chem. Int. Ed. 48, 7298 (2009).

${ }^{3}$ D. Écija, R. Otero, L. Sánchez, J. M. Gallego, Y. Wang, M. Alcamí, F. Martín, N. Martín, and R. Miranda, Angew. Chem. 119, 8020 (2007).

${ }^{4}$ J. Repp, G. Meyer, S. M. Stojković, A. Gourdon, and C. Joachim, Phys. Rev. Lett. 94, 026803 (2005).

${ }^{5}$ J. Repp, G. Meyer, and K. H. Rieder, Phys. Rev. Lett. 92, 036803 (2004).

${ }^{6}$ L. Gross, F. Mohn, N. Moll, P. Liljeroth, and G. Meyer, Science 325, 1110 (2009).

${ }^{7}$ L. Gross, Nat. Chem. 3, 273 (2011).

${ }^{8}$ O. Custance, R. Perez, and S. Morita, Nat. Nanotechnol. 4, 803 (2009).

${ }^{9}$ C. Bombis, N. Kalashnyk, W. Xu, E. Lægsgaard, F. Besenbacher, and T. R. Linderoth, Small 5, 2177 (2009).

${ }^{10}$ Ch. Wöll, S. Chiang, R. J. Wilson, and P. H. Lippel, Phys. Rev. B 39, 7988 (1989).

${ }^{11}$ W. Chen, V. Madhavan, T. Jamneala, and M. F. Crommie, Phys. Rev. Lett. 80, 1469 (1998).
${ }^{12}$ K. Schouteden, P. Lievens, and C. Van Haesendonck, Phys. Rev. B 79, 195409 (2009).

${ }^{13}$ I. Horcas, R. Fernandez, J. M. Gomez-Rodriguez, J. Colchero, J. Gomez-Herrero, and A. M. Baro, Rev. Sci. Instrum. 78, 013705 (2007).

${ }^{14}$ M. E. Cañas-Ventura, W. Xiao, P. Ruffieux, R. Rieger, K. Müllen, H. Brune, and R. Fasel, Surf. Sci. 603, 2294 (2009).

${ }^{15}$ K. Lauwaet, K. Schouteden, E. Janssens, C. Van Haesendonck, and P. Lievens (unpublished).

${ }^{16}$ W. Hebenstreit, J. Redinger, Z. Horozova, M. Schmid, R. Podloucky, and P. Varga, Surf. Sci. 424, L321 (1999).

${ }^{17}$ J. E. Inglesfield, J. Phys. C: Solid State Phys. 14, 3795 (1981).

${ }^{18}$ E. V. Chulkov, V. M. Silkin, and P. M. Echenique, Surf. Sci. 437, 330 (1999).

${ }^{19}$ L. Limot, T. Maroutian, P. Johansson, and R. Berndt, Phys. Rev. Lett. 91, 196801 (2003).

${ }^{20}$ R. Hoffmann, M. A. Lantz, H. J. Hug, P. J. A. van Schendel, P. Kappenberger, S. Martin, A. Baratoff, and H. J. Güntherodt, Appl. Surf. Sci. 188, 238 (2002).

${ }^{21}$ P. Varga and M. Schmid, Appl. Surf. Sci. 141, 287 (1999).

${ }^{22}$ R. Bechstein, C. Gonzàlez, J. Schütte, P. Jelínek, R. Pérez, and A. Kühnle, Nanotechnology 20, 505703 (2009). 
${ }^{23}$ L. R. Merte, L. C. Grabow, G. Peng, J. Knudsen, H. Zeuthen, W. Kudernatsch, S. Porsgaard, E. Lægsgaard, M. Mavrikakis, and F. Besenbacher, J. Phys. Chem. C 115, 2089 (2011).

${ }^{24}$ W. A. Hofer, Prog. Surf. Sci. 71, 147 (2003).

${ }^{25}$ J. P. Perdew, K. Burke, and M. Ernzerhof, Phys. Rev. Lett. 77, 3865 (1996).

${ }^{26}$ S. Grimme, J. Comput. Chem. 27, 1787 (2006).

${ }^{27}$ Within the standard DFT calculations, where the van der Waals interactions are not well described, the interaction of two- and threelayers-thick films with the $\mathrm{Au}(111)$ surface is extremely weak. The resulting interface distance is as large as $3.4 \AA$. The $\mathrm{NaCl}$-induced work function change, which is strongly dependent on the interface distance, is $0.4 \mathrm{eV}$, much lower than the experimental estimate.

${ }^{28}$ R. Bennewitz, A. S. Foster, L. N. Kantorovich, M. Bammerlin, C. Loppacher, S. Schär, M. Guggisberg, E. Meyer, and A. L. Shluger, Phys. Rev. B 62, 2074 (2000).
${ }^{29}$ J. Repp, G. Meyer, and K.-H. Rieder, Phys. Rev. Lett. 92, 036803 (2004).

${ }^{30}$ D. P. Woodruff, W. A. Royer, and N. V. Smith, Phys. Rev. B 34, 764 (1986).

${ }^{31}$ R. Courths, H. Wern, U. Hau, B. Cord, V. Bachelier, and S. Hüfner, J. Phys. F: Met. Phys. 14, 1559 (1984).

${ }^{32}$ R. Paniago, R. Matzdorf, G. Meister, and A. Goldmann, Surf. Sci. 336, 113 (1995).

${ }^{33}$ S. D. Kevan and R. H. Gaylord, Phys. Rev. B 36, 5809 (1987).

${ }^{34}$ D. Straub and F. J. Himpsel, Phys. Rev. B 33, 2256 (1986).

${ }^{35}$ C. Reuß, W. Wallauer, and Th. Fauster, Surf. Rev. Lett. 3, 1547 (1994).

${ }^{36}$ N. W. Ashcroft and N. D. Mermin, Solid State Physics (Holt, Rinehart and Winston, New York, 1976), Vol. 826. 